\title{
Molecular grading of tumors of the upper urothelial tract using FGFR3 mutation status identifies patients with favorable prognosis
}

This article was published in the following Dove Press journal:

Research and Reports in Urology

30 November 2012

Number of times this article has been viewed

Stephen R Lyle'

Chung-Cheng Hsieh'

Cecilia A Fernandez ${ }^{2}$

Anthony P Shuber ${ }^{2}$

'University of Massachusetts, Worcester, MA, ${ }^{2}$ Predictive

Biosciences Inc., Lexington, MA, USA

Correspondence: Stephen R Lyle University of Massachusetts Medical

School, 364 Plantation St, LRB

Worcester, MA 01605, USA

Tel +l 5088654774

Email stephen.lyle@umassmed.edu
Background: Mutations in FGFR3 have been shown to occur in tumors of the upper urothelial tract and may be indicative of a good prognosis. In bladder tumors, the combination of FGFR3 mutation status and Ki-67 level has been used to define a tumor's molecular grade and predict survival. Pathological evaluation of upper urothelial tumors is currently the best predictor of prognosis, but suffers from variability in pathological assessments. This study investigated the association with prognosis of FGFR3 mutations alone and in combination with $\mathrm{Ki}-67$ in this patient population.

Methods: Genomic DNA was isolated from tumor samples of 80 patients with upper urothelial cancer. The presence of mutation in FGFR 3 was evaluated using real-time polymerase chain reaction. Ki-67 protein expression was determined by immunohistochemistry. Kaplan-Meier survival analysis evaluated the relationship of FGFR3 mutations and Ki-67 levels with survival.

Results: FGFR 3 mutations were identified in $40 \%$ of tumors and were predominantly associated with noninvasive tumors. Overall survival was higher in patients with FGFR3 mutant tumors $(P=0.02)$ and in molecular grade 1 tumors as determined by $F G F R 3$ and Ki-67 $(P=0.02)$.

Conclusion: In this study, we confirm the occurrence of FGFR 3 mutations in tumors of the upper urothelial tract and its association with a good prognosis. Both FGFR3 and molecular grading are predictors of overall survival. Molecular grading can help to assess the prognosis of patients with upper urinary tract cancer and may represent a new tool for managing this population of patients.

Keywords: upper tract, urothelial carcinoma, ureter, renal pelvis, Ki-67, survival

\section{Background}

Urothelial cancer is the seventh leading cause of cancer in the US. ${ }^{1}$ The majority of urothelial cancers occur in the bladder, with only about $5 \%-10 \%$ occurring in the upper urinary tract. ${ }^{2,3}$ About $75 \%$ of upper urinary tract tumors are found in the collecting system of the kidney, with the remainder being found in the ureter. ${ }^{4}$

Activating mutations in the fibroblast growth factor receptor 3 gene (FGFR3) are one of the most frequent somatic mutations found in bladder cancer..$^{5-7}$ Eight FGFR3 mutations in three exons (exons 7, 10, and 15) are associated with $>90 \%$ of all known mutant FGFR3-positive bladder cancers and are also common in upper urinary tract cancers. ${ }^{8,9}$ FGFR 3 mutations occur in about $50 \%$ of primary bladder and upper tract tumors and are associated with low-stage tumors of the bladder and ureter. ${ }^{5-7,9-11}$ FGFR3 mutations are also associated with a milder disease course in both bladder and upper urothelial cancers, as well as better survival in patients with invasive urothelial cancer of the upper tract. ${ }^{9}$ 
Pathological evaluation of upper urothelial tumors is currently the best predictor of prognosis, but suffers from variability in pathological assessments. ${ }^{12-16} \mathrm{~A}$ number of studies have now shown that for bladder tumors, the combination of FGFR3 and Ki-67 staining can be used to increase accuracy in grading and can be indicative of prognosis. Ki-67 is a nuclear protein that is highly expressed in proliferating cells and has been shown to be associated with long-term survival in tumors of the upper tract. ${ }^{17}$ In this study, we used both FGFR3 mutational analysis and immunohistochemistry for the Ki-67 antigen to evaluate the prognosis of patients with upper urothelial cancer.

\section{Materials and methods Experimental samples}

Eighty formalin-fixed, paraffin-embedded archived patient tissue samples of urothelial carcinoma from the upper urinary tract were obtained from the UMass Cancer Center Tissue Bank with institutional review board approval. Of these, 28 tumors were localized to the ureter and 52 were localized to the renal pelvis. Each case was reviewed by a pathologist to confirm stage and morphological grade. Baseline patient demographics are shown in Table 1.

\section{DNA isolation from urothelial tumor samples}

DNA was isolated from three pooled $5 \mu \mathrm{m}$ sections of formalin-fixed, paraffin-embedded upper urothelial tumor tissue using the Qiagen QIAamp DNA FFPE tissue kit (Qiagen, Valencia, CA) as per the manufacturer's instructions, except for the following modifications. During removal of the paraffin, the samples were centrifuged for a further 10 minutes

Table I Baseline demographics and disease characteristics by evidence of disease

\begin{tabular}{ll}
\hline Characteristics, $\mathrm{n}(\%)$ & $\mathrm{n}=80$ \\
Gender & \\
Male & $44(55)$ \\
Median age, years (range) & $72(43-84)$ \\
TNM staging & \\
Ta & $29(36.3)$ \\
T1 & $11(13.8)$ \\
T2 & $6(7.5)$ \\
T3 & $32(40)$ \\
T4 & $2(2.5)$ \\
Grade & \\
Low & $17(21.3)$ \\
High & $63(78.8)$ \\
Site & \\
Ureter & $28(35)$ \\
Renal pelvis & $52(65)$ \\
\hline
\end{tabular}

following treatment with xylene and 100\% ethanol washes. In addition, samples were incubated with proteinase $\mathrm{K}$ for 16 hours prior to processing further to ensure complete digestion of tissue.

\section{Detection of FGFR3 mutations}

Tumor genomic DNA was amplified in a multiplex, realtime polymerase chain reaction (PCR) with primers and dual-labeled fluorescent probes specific for human FGFR3 exons 7, 10, and 15 using the Roche LightCycler 480 (Roche Applied Sciences, Indianapolis, IN) under standard conditions and as previously described by us. ${ }^{18}$ PCR conditions were adjusted to account for a lower DNA yield and DNA degradation due to formalin fixation. FastStart Taq polymerase, dNTP mix, and reaction buffers were sourced from Roche Applied Science. PCR products were purified using ExoSAP-IT (Affymetrix/USB, Cleveland, $\mathrm{OH}$ ). Oligonucleotide primers and fluorescently labeled probes were designed using the Oligo 7.0 program (MBI Inc, Cascade, CO). All primers were synthesized by Integrated DNA Technologies Inc (Coralville, IA).

A second multiplexed PCR was carried out to identify FGFR3 mutations. This PCR reaction used primers that encoded wild-type sequence with locked nucleic acid (LNA) bases surrounding and including a known mutation site in exon 7, 10, or 15 (exon 7 [R248C and S249C], exon 10 [G372C, S373C and Y375C], and exon 15 [K652E, K652M, K652T and K652Q]), and real-time PCR primers specific for each exon with dual-labeled fluorescent probes. ${ }^{18}$ Because the LNA primers have a higher annealing affinity with wild-type DNA than with mutant DNA, they promote amplification of mutant DNA by inhibiting wild-type DNA amplification. All PCR experiments were carried out in duplicate using the Roche LightCycler 480.

As a control for the amount of DNA included in each reaction, PCR amplification was performed in the absence of LNA-containing primers. A ratio between the amplification cycles of positive controls (plasmids containing known FGFR3 mutations) and negative controls (wild-type DNA) were included in each assay to determine if a sample did or did not contain a mutation. Both positive and negative control plasmids were diluted with human genomic DNA to a final concentration of $1 \%$. All samples were found to have sufficient DNA for analysis.

\section{Ki-67 immunohistochemistry}

One adjacent $5 \mu \mathrm{m}$ section of the formalin-fixed, paraffinembedded upper urothelial tumor tissue used for mutation 
detection was used to assess Ki-67 expression. Tumor tissues were processed using Ki-67-specific monoclonal antibodies (Ventana clone MM1) as per the manufacturer's protocol on a Ventana Benchmark XT Autostainer. Ki-67 level scores were established from three independent fields of the highest level of staining per slide. Samples were classified as high for $\mathrm{Ki}-67$ if $\geq 25 \%$ cells per field were positive for $\mathrm{Ki}-67$ and as low if $<25 \%$ cells per field were positive for Ki-67 staining.

\section{Molecular grading}

Molecular grade was defined as previously described. ${ }^{10,11}$ In brief, molecular grade 1 is defined as a sample that is positive for an FGFR3 mutation and has low Ki-67 staining. Molecular grade 2 is defined as samples that are positive for an FGFR3 mutation but have high Ki-67 staining, or are wild-type for FGFR3 and have low Ki-67 staining. Molecular grade 3 is defined as a sample that has wild-type FGFR3 and high Ki-67 staining.

\section{Statistical analysis}

Overall survival was measured from the date of first cancer diagnosis to the date of death from any cause and was censored from the date of last follow-up for survivors. Overall survival probability was estimated by the Kaplan-Meier method for each patient group classified by the FGFR3 mutation status or classified according to the molecular grade defined by the combination of FGFR3 and Ki-67. Differences in survival probabilities among patient groups were evaluated with use of the log-rank test. A two-sided $P$ value of less than 0.05 was considered to indicate statistical significance. The Cox proportional hazard model was used to assess the simultaneous contribution of the following baseline covariates: age, gender, tumor stage, morphological grade, site, FGFR3 mutation status, Ki-67, and molecular grade. REMARK (REporting recommendations for tumour MARKer prognostic studies) guidelines were used for this study. ${ }^{19}$

\section{Results}

\section{FGFR3 mutations in tumors of upper urothelial origin}

Although many studies have shown that FGFR3 mutations are common in bladder cancer, only one other study has shown that tumors of upper urothelial origin can also harbor FGFR3 mutations. In this study, 80 tumors from patients with upper urothelial cancer were analyzed for mutations in FGFR3. Most tumors were of high morphological grade, and either stages Ta or T3, and were removed from the renal pelvis region (Table 1). Of the 80 patients, 32 had tumors with FGFR3 mutations, indicating a mutation frequency of $40 \%$ (Table 2). The frequency in the ureter and renal pelvis was $50 \%$ and $34 \%$, respectively. FGFR3 mutations were more frequent in noninvasive tumors (53\% [21/40]) compared with $28 \%$ [21/40] in invasive tumors and were associated with low-grade disease (71\% [12/17] of low-grade tumors carried mutations compared with $32 \%$ [20/63] of high-grade tumors), regardless of tumor site and in contrast with previous studies. ${ }^{9}$

\section{FGFR3 mutations and molecular grade are associated with increased survival}

Evaluating urothelial cancer prognosis by pathology stage and grade has poor reproducibility and is a major concern for clinicians. Morphological grade assessment may differ in about $50 \%$ of cases. ${ }^{14,15,20}$ There is a clinical need for developing assays that are useful both for diagnosing and for assessing the prognosis of upper urinary tract cancer. Therefore, we analyzed survival as a function of FGFR3 mutation status using Kaplan-Meier survival plots. Patients whose tumors contained an FGFR3 mutation had better overall survival than those whose tumors did not have a mutation (Figure 1, $P=0.02$ ), consistent with previous findings. ${ }^{9}$ From Cox proportional hazard analysis, patients with wild-type $F G F R 3$ had approximately two times higher mortality than patients with FGFR 3 mutations (hazards ratio $1.95,95 \%$ confidence interval $0.70-5.45$ ) after adjusting for stage and other covariates. The hazards ratio estimate did not reach statistical significance $(P=0.20)$, and a larger

Table 2 Frequency of FGFR3 mutations and Ki-67 staining

\begin{tabular}{|c|c|c|}
\hline & Frequency, n (\%) & $95 \% \mathrm{Cl}$ \\
\hline \multicolumn{3}{|l|}{ FGFR3 mutations } \\
\hline $\begin{array}{l}\text { All upper urothelial tract } \\
(\mathrm{n}=80)\end{array}$ & $32 / 80(40)$ & $29-52$ \\
\hline Ureter $(n=28)$ & $14 / 28(50)$ & $31-69$ \\
\hline Renal pelvis $(n=52)$ & $18 / 52(34)$ & $22-49$ \\
\hline \multicolumn{3}{|l|}{ Stage } \\
\hline $\mathrm{Ta}$ & I6/29 (55) & $36-74$ \\
\hline TI & $5 / 11(45)$ & I7-77 \\
\hline$\geq \mathrm{T} 2$ & I I/40 (27) & $15-44$ \\
\hline \multicolumn{3}{|l|}{ Pathology grade } \\
\hline Low & $12 / 17(7 \mid)$ & $44-90$ \\
\hline High & $20 / 63(32)$ & $21-45$ \\
\hline \multicolumn{3}{|l|}{ Ki-67 } \\
\hline Low & $58 / 80(73)$ & $6|-8|$ \\
\hline High & $22 / 80(27)$ & $18-39$ \\
\hline
\end{tabular}

Abbreviation: $\mathrm{Cl}$, confidence interval. 


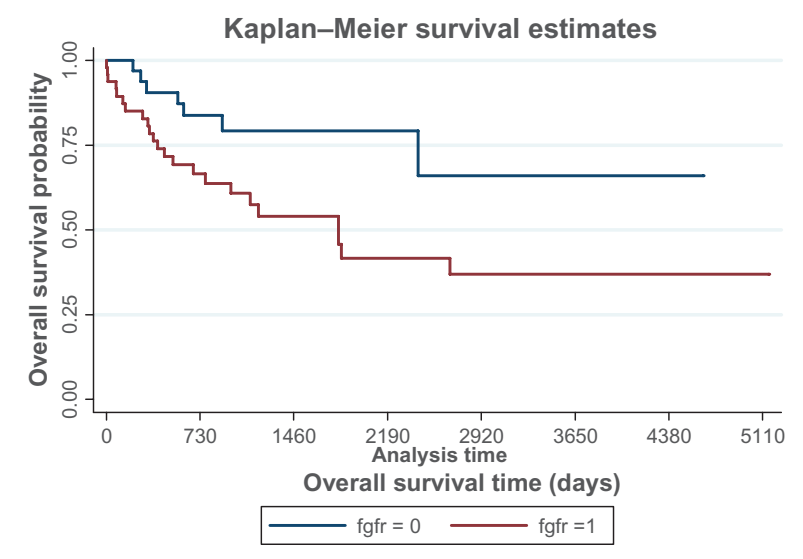

Figure I Kaplan-Meier analysis for survival estimates by FGFR3 mutational status, where 0 is mutant and I is wild-type.

Notes: Statistical significance was determined by log-rank test. FGFR3 was associated with improved overall survival $(P=0.02)$.

set of samples would be needed to confirm if FGFR3 is an independent predictor of overall survival.

In addition, we explored whether the combination of FGFR3 and Ki-67 might be a better predictor of prognosis than pathological grade alone. The molecular grade established by FGFR3 and Ki-67 was defined as described in previous studies. ${ }^{10,11}$ Table 3 describes the distribution of molecular grade based on the combination of FGFR3 and Ki-67. Although Ki-67 levels showed some association with survival, the log-rank test results were not significant (data not shown). However, patients with molecular grade 1 tumors had significantly better survival estimates than those with molecular grade 2 or 3 tumors $(P=0.02$, Figure 2$)$. The results of the log-rank test demonstrate that molecular grade is a predictor of survival. Molecular grade 1 tumors (as assessed by FGFR3 and Ki-67) are associated with better survival compared with wild-type FGFR3 and/or increased Ki-67 expression.

\section{Discussion}

Previous studies have shown that FGFR3 mutations are associated with an improved prognosis in invasive tumors

Table 3 Distribution by molecular grade

\begin{tabular}{lll}
\hline & Frequency, $\mathbf{n}(\%)$ & $\mathbf{9 5 \%} \mathbf{~ I ~}$ \\
\hline MG I & & \\
Low Ki-67/mutant FGFR3 & $29 / 80(36)$ & $26-48$ \\
MG2 & & \\
Low Ki-67/wild-type FGFR3 & $29 / 80(36)$ & $26-48$ \\
High Ki-67/mutant FGFR3 & $3 / 80(4)$ & $1-10$ \\
MG3 & & \\
High Ki-67/wild-type FGFR3 & $19 / 80(24)$ & $15-35$ \\
\hline
\end{tabular}

Abbreviations: MG, molecular grade; $\mathrm{Cl}$, confidence interval.

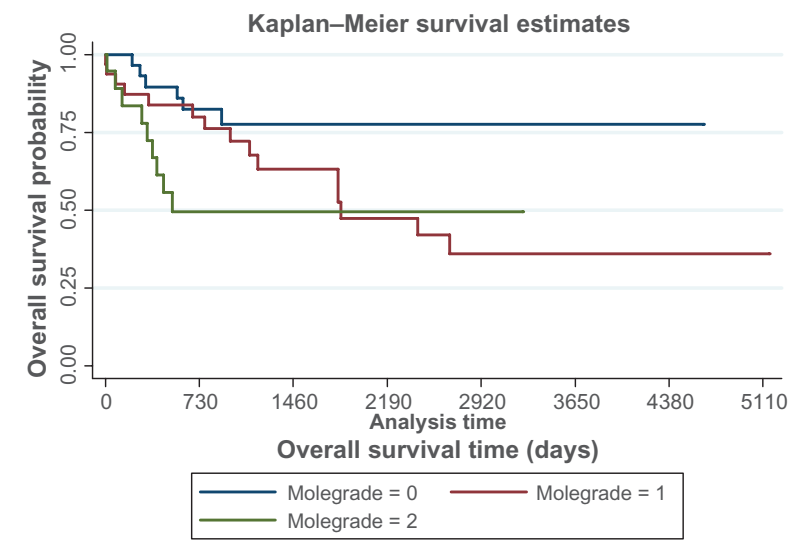

Figure $\mathbf{2}$ Kaplan-Meier analysis for survival estimates by molecular grade, where 0 is molecular grade I, I is molecular grade 2 and 2 is molecular grade 3.

Notes: Statistical significance was determined by log-rank test. Molecular grade was associated with improved overall survival $(P=0.02)$.

of the upper urinary tract, while increased Ki67 levels have been associated with a poor prognosis. ${ }^{9,17}$ Other studies have shown that the combination of FGFR3 and Ki67 can be used to determine the "molecular grade" of bladder cancer tumors. ${ }^{10}$ In this study, we confirm the association of FGFR3 mutations with improved prognosis in a cohort of both invasive and noninvasive urothelial tumors of the upper urinary tract and apply the use of molecular grade to determine whether this approach can be indicative of a good prognosis. The results of our FGFR3 mutation assay are consistent with other methods reported to have similar analytical sensitivity. ${ }^{8}$ Molecular grading provides an objective method for determining tumor grade and eliminates the variability of pathological evaluation.

Both FGFR3 mutation and low molecular grade were associated with improved survival. Interestingly, of the 29 tumors in the molecular grade 1 group, 19 had previously been classified as "high grade". Of these, 14 (74\%) were alive as of the date of this study, ie, an average of 2000 days (5.7 years) post diagnosis and treatment, whereas the 5-year survival of high morphological grade tumors has been shown to be as low as $28 \% .{ }^{21}$ These results support the use of molecular grading of tumors of the upper urinary tract to grade tumors more accurately and predict prognosis. These findings are consistent with prior results that found that the combination of FGFR3 and $\mathrm{Ki}-67$ were good predictors of bladder cancer prognosis. ${ }^{10,11}$

Given its known association with bladder cancer, the FGFR3 mutation detection assay has been developed as a diagnostic tool for both molecular grading of bladder cancer tissues and for urine-based assays used in the evaluation of patients with hematuria or for cancer recurrence in patients 
who had been previously treated for bladder cancer. ${ }^{18,22}$ In the urine-based assay, FGFR 3 has been shown to have very high specificity $(>95 \%)$ and a positive predictive value. ${ }^{18}$ It is possible that detection of FGFR3 mutations in urine may also be useful in diagnosing cancer of the upper urinary tract. In fact, a recent report showed that in a community-based clinical setting, FGFR3 mutation analysis was used to diagnose an upper urothelial carcinoma in a patient in whom standard diagnostic methods were inconclusive. ${ }^{23}$

The results of this study are consistent with previous findings that FGFR 3 mutations are present in tumors of the upper urinary tract, and are indicative of a good prognosis. We further demonstrate that molecular grading using FGFR3 and Ki-67 can accurately grade tumors and may represent a new, powerful tool for managing this population of patients.

\section{Acknowledgments}

The authors would like to thank Lei Li and Carol Ahern for technical assistance, Karen Dresser for immunohistochemical staining, and Elizabeth Goodwin for editorial assistance.

\section{Disclosure}

SRL and CCH report no conflicts of interest in this work. APS and CAF are employees of Predictive Biosciences.

\section{References}

1. Jemal A, Bray F, Center MM, Ferlay J, Ward E, Forman D. Global cancer statistics. CA Cancer J Clin. 2011;61(2):69-90.

2. Chow WH, Dong LM, Devesa SS. Epidemiology and risk factors for kidney cancer. Nat Rev Urol. 2010;7(5):245-257.

3. Roupret M, Zigeuner R, Palou J, et al. European guidelines for the diagnosis and management of upper urinary tract urothelial cell carcinomas: 2011 update. Eur Urol. 2011;59(4):584-594.

4. Hall MC, Womack S, Sagalowsky AI, Carmody T, Erickstad MD, Roehrborn CG. Prognostic factors, recurrence, and survival in transitional cell carcinoma of the upper urinary tract: a 30 -year experience in 252 patients. Urology. 1998;52(4):594-601.

5. Billerey C, Chopin D, Aubriot-Lorton MH, et al. Frequent FGFR3 mutations in papillary non-invasive bladder (pTa) tumors. Am J Pathol. 2001;158(6):1955-1959.

6. Cappellen D, De Oliveira C, Ricol D, et al. Frequent activating mutations of FGFR3 in human bladder and cervix carcinomas. Nat Genet. 1999;23(1):18-20.

7. van Rhijn BW, Lurkin I, Radvanyi F, Kirkels WJ, van der Kwast TH, Zwarthoff EC. The fibroblast growth factor receptor 3 (FGFR3) mutation is a strong indicator of superficial bladder cancer with low recurrence rate. Cancer Res. 2001;61(4):1265-1268.

Research and Reports in Urology

\section{Publish your work in this journal}

Research and Reports in Urology is an international, peer-reviewed, open access journal publishing original research, reports, editorials, reviews and commentaries on all aspects of adult and pediatric urology in the clinic and laboratory including the following topics: Pathology, pathophysiology of urological disease; Investigation and treatment of
8. van Oers JM, Lurkin I, van Exsel AJ, et al. A simple and fast method for the simultaneous detection of nine fibroblast growth factor receptor 3 mutations in bladder cancer and voided urine. Clin Cancer Res. 2005;11(21):7743-7748.

9. van Oers JM, Zwarthoff EC, Rehman I, et al. FGFR3 mutations indicate better survival in invasive upper urinary tract and bladder tumours. Eur Urol. 2009;55(3):650-657.

10. van Rhijn BW, Vis AN, van der Kwast TH, et al. Molecular grading of urothelial cell carcinoma with fibroblast growth factor receptor 3 and MIB- 1 is superior to pathologic grade for the prediction of clinical outcome. J Clin Oncol. 2003;21(10):1912-1921.

11. van Rhijn BW, Zuiverloon TC, Vis AN, et al. Molecular grade (FGFR3/ MIB-1) and EORTC risk scores are predictive in primary non-muscleinvasive bladder cancer. Eur Urol. 2010;58(3):433-441.

12. Allard P, Bernard P, Fradet Y, Tetu B. The early clinical course of primary Ta and T1 bladder cancer: a proposed prognostic index. Br J Urol. 1998;81(5):692-698.

13. Millan-Rodriguez F, Chechile-Toniolo G, Salvador-Bayarri J, HuguetPerez J, Vicente-Rodriguez J. Upper urinary tract tumors after primary superficial bladder tumors: prognostic factors and risk groups. $J$ Urol. 2000;164(4):1183-1187.

14. Ooms EC, Anderson WA, Alons CL, Boon ME, Veldhuizen RW. Analysis of the performance of pathologists in the grading of bladder tumors. Hum Pathol. 1983;14(2):140-143.

15. Tosoni I, Wagner U, Sauter G, et al. Clinical significance of interobserver differences in the staging and grading of superficial bladder cancer. BJU Int. 2000;85(1):48-53.

16. Vikram R, Sandler CM, Ng CS. Imaging and staging of transitional cell carcinoma: part 2, upper urinary tract. AJR Am J Roentgenol. 2009;192(6):1488-1493.

17. Rey A, Lara PC, Redondo E, Valdes E, Apolinario R. KI67 proliferation index in tumors of the upper urinary tract as related to established prognostic factors and long-term survival. Arch Esp Urol. 1998;51(2):204-210.

18. Karnes RJ, Fernandez CA, Shuber AP. A non-invasive multi-analyte urine based diagnostic assay for urothelial cancer of the bladder in the evaluation of hematuria. Mayo Clin Proc. 2012;87(9):835-842.

19. McShane LM, Altman DG, Sauerbrei W, Taube SE, Gion M, Clark GM. REporting recommendations for tumor MARKer prognostic studies (REMARK). Breast Cancer Res Treat. 2006;100(2):229-235.

20. van Rhijn BW, Lurkin I, Chopin DK, et al. Combined microsatellite and FGFR3 mutation analysis enables a highly sensitive detection of urothelial cell carcinoma in voided urine. Clin Cancer Res. 2003;9(1):257-263.

21. Lee SH, Lin JS, Tzai TS, et al. Prognostic factors of primary transitional cell carcinoma of the upper urinary tract. Eur Urol. 1996;29(3):266-270.

22. Fernandez CA, Millholland JM, Zwarthoff EC, Feldman AS, Karnes RJ, Shuber AP. A noninvasive multi-analyte diagnostic assay: combining protein and DNA markers to stratify bladder cancer patients. Research and Reports in Urology. 2012;4:17-26.

23. Silverberg D. Urothelial carcinoma of the upper urinary tract diagnosed via FGFR3 mutation detection in urine: a case report. BMC Urol. 2012;12(1):20.

urological disease; Pharmacology of drugs used for the treatment of urological disease. The manuscript management system is completely online and includes a very quick and fair peer-review system, which is all easy to use. Visit http://www.dovepress.com/testimonials.php to read real quotes from published authors. 